\title{
Long-Lived Electron Capture Dissociation Product Ions Experience Radical Migration via Hydrogen Abstraction
}

\author{
Peter B. O'Connor, Cheng Lin, Jason J. Cournoyer, Jason L. Pittman, \\ Marina Belyayev, and Bogdan A. Budnik \\ Mass Spectrometry Resource, Department of Biochemistry, Boston University School of Medicine, Boston, \\ Massachusetts, USA
}

\begin{abstract}
To explore the mechanism of electron capture dissociation (ECD) of linear peptides, a set of 16-mer peptides were synthesized with deuterium labeled on the $\alpha$-carbon position of four glycines. The ECD spectra of these peptides showed that such peptides exhibit a preference for the radical to migrate to the $\alpha$-carbon position on glycine via hydrogen (or deuterium) abstraction before the final cleavage and generation of the detected product ions. The data show c-type fragment ions, ions corresponding to the radical cation of the c-type fragments, c., and they also show c--1 peaks in the deuterated peptides only. The presence of the c-1 peaks is best explained by radical-mediated scrambling of the deuterium atoms in the long-lived, metastable, radical intermediate complex formed by initial electron capture, followed by dissociation of the complex. These data suggest the presence of at least two mechanisms, one slow, one fast. The abundance of $\mathrm{H} \cdot$ and - $\mathrm{CO}$ losses from the precursor ion changed upon deuterium labeling indicating the presence of a kinetic isotope effect, which suggests that the values reported here represent an underestimation of radical migration and $\mathrm{H} / \mathrm{D}$ scrambling in the observed fragments. (J Am Soc Mass Spectrom 2006, 17, 576-585) (c) 2006 American Society for Mass Spectrometry
\end{abstract}

$\mathrm{E}$ lectron capture dissociation (ECD) [1, 2] generates fragmentation patterns that are reminiscent of odd-electron EI fragmentation patterns from small molecules [3], but occur at lower internal vibrational energy [4]. This is advantageous because there is insufficient energy to break hydrogen bonds and noncovalent interactions in the gas phase, even though the backbone has been cleaved allowing study of protein $3 \mathrm{D}$ structure in the gas phase [5]. Furthermore, ECD preferentially cleaves backbone $\mathrm{N}-\mathrm{C}_{\alpha}$ bonds over sidechain bonds, allowing determination of the exact sites of labile side-chain modifications [6-9]. Such behavior has been suggested to be evidence of a nonergodic dissociation pathway [1], but there is also a body of calculations that suggests that nonergodicity may not be required to explain the observed fragments [10-13].

In a previously published manuscript [14], data were presented in which cyclic peptides were shown to undergo extensive secondary side-chain and backbone fragmentation that appeared to be free-radical driven. Secondary side-chain fragmentation has also been reported by other research groups [15-19], and has been explored by a combined experimental and computa-

Published online February 28, 2006

Address reprint requests to Professor P. B. O'Connor, Mass Spectrometry Resource, Department of Biochemistry, Boston University School of Medicine, 670 Albany St., Rm. 507, Boston, MA 02118, USA. E-mail: poconnor@ bu.edu tional approach as well [20]. One significant limitation in the cyclic peptide data, however, is that it is derived from molecules for which primary backbone cleavages do not result in mass shifts and, thus, are unobservable. While these data were helpful for determining the existence of secondary backbone fragmentation in ECD, such data cannot provide information concerning which fragments are generated from primary versus secondary cleavages. The cyclic peptide data, however, did suggest a fragmentation pathway in which the expected $\mathrm{c} / \mathrm{z}$ ion cleavages could arise both from a primary nonergodic fragmentation and also from a secondary ergodic pathway. If primary and secondary fragments in ECD could be distinguished, it would be possible to determine if the secondary free-radical rearrangements do, indeed, result in c/z ions, and thereby obtain information that would show if long-lived radical intermediates are important in the ECD mechanism.

The free radical cascade mechanism [14] requires that the radical(s) formed from the primary fragmentation have time to migrate before the final, observed fragmentation. This is a process that is expected to occur on the nanosecond to microsecond time scale, which is much longer than the expected time frame for intramolecular vibrational redistribution (picoseconds) $[21,22]$. If the free radical cascade mechanism is correct, previous calculations $[10,11,23,24]$ suggested that it would preferentially migrate to the alpha carbon posi- 
tion via hydrogen abstraction, and that there would be a preference for migration to glycine because the lack of steric hindrance would allow a radical in that position to become planar with the carbonyl [23]. This hydrogen abstraction reaction typically proceeds via a low-energy barrier transition state and is even exothermic in some cases [24]. Hence, the alpha carbon position is expected to be critical in most of the proposed reaction schemes [14]. Thus, an experiment was proposed in which a peptide, deuterium labeled at several amino acid $\alpha$ carbon positions, would be subjected to ECD fragmentation. If $\alpha$-carbon hydrogen abstraction were occurring, deuterium scrambling would result and would be detectable in the mass spectrum as $\pm 1.006 \mathrm{Da}$ mass shifts, $\pm(+\mathrm{D}-\mathrm{H})$, in the isotopic distributions of the fragment ions.

To test this hypothesis, two pairs of peptides were synthesized. In each pair, both peptides had the same sequence which included four glycine residues, but one peptide had the glycines doubly deuterium labeled on the alpha carbons. Comparisons of the electron capture dissociation spectra of each pair of peptides show strong differences in the isotopic pattern, suggesting that the radical does generally migrate to the alpha carbon position of glycine.

\section{Experimental}

The mass spectrometer used was a previously described $[25,26]$ electrospray ionization Fourier transform mass spectrometer. The four peptides, listed in Table 1, were synthesized by Anaspec (San Jose, CA). The peptides were dissolved to $\sim 1 \mathrm{mM}$ in $\mathrm{H}_{2} \mathrm{O}$, aliquoted into 10 separate vials, and stored at $-20^{\circ} \mathrm{C}$. Immediately before use, an aliquot was thawed, diluted to $\sim 1 \mathrm{pmol} / \mu \mathrm{l}$ in 50:50:1 $\mathrm{H}_{2} \mathrm{O}: \mathrm{CH}_{3} \mathrm{OH}$ :formic acid, and $\sim 1-5 \mu \mathrm{L}$ of this solution was used in pulled glass capillary nanospray tips [27] to generate multiply charged ions. The lowenergy electrons needed for ECD were generated using an indirectly heated dispenser cathode [28] (model STD200, Heatwave, Watsonville, CA, heated with $5 \mathrm{~V}$ and $1.1 \mathrm{~A})$. ECD was preformed as previously described, [14] except that high-energy ECD [18] experiments were also performed. The center potential of the dispenser cathode was biased at either -0.25 or $-9 \mathrm{~V}$ relative to ground, with the grid at $+10 \mathrm{~V}$, the outer trapping plates held at $+10 \mathrm{~V}$, and the inner trapping ring held at $+1 \mathrm{~V}$ during ECD. The ECD experiments, which included monoisotopic isolation, used $0.8 \mathrm{eV}$ electrons. The duration of the ECD event varied from $15-50 \mathrm{~ms}$, but timing of the event had to be adjusted for

Table 1. Synthetic peptides used in these experiments

\begin{tabular}{ll}
\hline BUSM 1 & RAAAGADGDGAGADAR \\
BUSM 2 & RAAAG**ADG**DG**AG**ADAR \\
BUSM 3 & RAGADGDADGDAGAAR \\
BUSM 4 & RAG**ADG**DADG**DAG**AAR \\
\hline
\end{tabular}

*Indicates deuteration on the alpha carbon. best ECD efficiency because of periodic overlap between the electron beam and the ion cloud due to magnetron motion.

The spectra were acquired with a Nyquist frequency corresponding to a low $\mathrm{m} / \mathrm{z}$ limit of $\sim 215$, zerofilled twice, and Fourier transformed without apodization. Internal calibration on the $[\mathrm{M}+2 \mathrm{H}]^{2+}$ and $[\mathrm{M}+\mathrm{H}]^{+}$ peaks and their isotopes yielded a typical mass accuracy of 1-2 ppm. Greater than $95 \%$ of the ions in the spectra were identified, and corresponded to previously reported side-chain losses and the $a_{n}, b_{n}, c_{n}, y_{n}$, and $z_{n}$ ion series; no $d_{n}, w_{n}$ or $x_{n}$ ions were observed (following the Roepstorff and Fohlman nomenclature as modified by Biemann) [29, 30].

The peptide sequences, Table 1, were designed as follows. To maximize detection of the fragments, arginine was placed on each end. Also, as mentioned above, calculations [10,11, 23, 24] suggested that glycine was the preferential site of hydrogen abstraction by a radical because of the ability of a glycine radical to form a planar structure with the adjacent carbonyl stabilizing it by resonance. Furthermore, glycine has two hydrogens on the alpha carbon, thus doubling the probability of detecting a deuterium migration away from this site. Finally, the peptide was also designed with aspartic acid residues so that it could also be used as a control for other experiments designed to differentiate aspartic acid and isoaspartic acid [31-33].

\section{Results}

The results presented were compiled from a number of experiments: low-energy ECD $(0-1 \mathrm{eV})$, high-energy ECD $(\sim 9 \mathrm{eV}), \mathrm{Q} 2-\mathrm{CAD}$, SORI-CAD, and ECD of the monoisotopically isolated precursor. A typical ECD spectrum of these peptides is shown in Figure 1a. In this case, the spectrum was obtained with $0.8 \mathrm{eV}$ electrons and yielded $13 / 16$ of the possible c-ion cleavages, and $\sim 50 \%$ of the possible a and $\mathrm{z}$ ions. In almost all $\mathrm{c}_{\mathrm{n}}, \mathrm{z}_{\mathrm{n}}$, and $a_{n}$ isotopic distributions detected, both the radical and even electron form of the assigned cleavages were observed. This resulted in a typical isotopic pattern (Figure 1a inset), which corresponded to (at least) three overlapped components, one for the even electron fragment, one for the radical fragment, (which is $1 \mathrm{Da}$ lighter), and one which is $1.0078 \mathrm{Da}$ lighter than the radical fragment (named $c_{n} \cdot-1$ ). This $c_{n} \cdot-1$ peak appears only in the spectra of the deuterated peptides with implications discussed below.

Figure $1 \mathrm{~b}$ was the original spectrum which was acquired with the electron gun mounted on the trapping plate of the ICR cell, thus heating it to a high, but undetermined temperature. The presence of the $b_{7} / y_{7}$ and $b_{9} / y_{9}$ ions as well as the slight preference for the lower molecular weight $c$ ions is indication that the ions were somewhat vibrationally excited before or during the ECD experiment due to blackbody IR heating [34] of the ions from the hot cell, making this experiment a variant of the "activated-ion" ECD experiment [35]. Because of this, the 


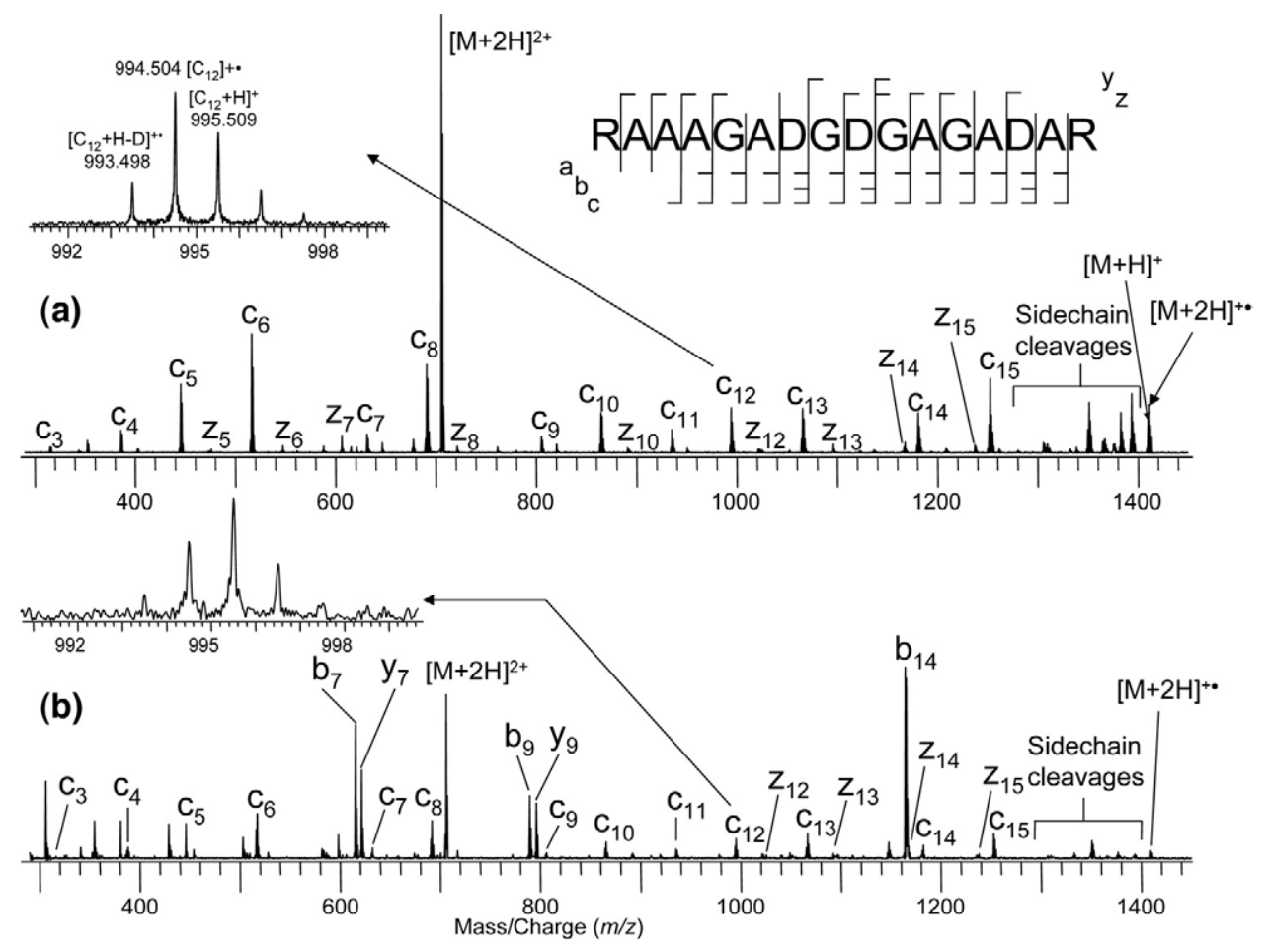

Figure 1. ECD mass spectra of BUSM 2 under (a) $0.25 \mathrm{eV}$ and (b) $9 \mathrm{eV}$ ECD conditions.

ICR cell was modified, and the electron gun was mounted $\sim 15 \mathrm{~cm}$ away from the cell with no direct attachment to the cell, and the cell plates were polished to a mirror shine. This eliminated all conductive and most radiative heating of the ions inside the cell. Figure 1a is the result. This spectrum shows strongly reduced abundance of the $b / y$ ion pairs mentioned above, and the $c$ ion series is shifted slightly to the right (higher mass), indicating lower total activation.

There is $\sim 10 \%$ under-deuteration of the precursor ion (data not shown), which is roughly evenly distributed among the eight deuterium labels as is apparent in the $b_{7} / y_{7}$ and $b_{9} / y_{9}$ ion pairs (from the initial mass spectrum without specific dissociation). The under-deuteration amount is the same for both deuterated peptides, implying a $\sim 97.5 \%$ purity in the $\mathrm{d}_{2}$-glycine synthesis precursor. Thus, it is important to deduct $\sim 2.5 \%$ /glycine from the abundance of the deuterium-loss peak, $[\mathrm{M}+\mathrm{H}-\mathrm{D}]$, before interpretation of the data with respect to radical driven deuterium scrambling. All peptide deuterium scrambling percentages reported in this paper are ratios of the $c_{n} \cdot-1$ to $c_{n} \cdot$ ions, and all reported deuterium scrambling ratios have been corrected for the under-deuteration as mentioned above.

To determine whether or not H/D scrambling was occurring in solution before the various MS experiments, one aliquot of the deuterium labeled peptides was simply left on the bench at $\mathrm{pH} 7$ for two weeks. When the ECD spectra of these "aged" samples were run, the extent of under-deuteration was unchanged, as were the abundances of the c-1 ion peaks. Thus, for these peptides at least, the alpha carbon positions of glycine are not exchangeable under such conditions. Generally, as discussed above, the peptides shown were treated much more carefully with the aliquots stored in water (ice) at $-20{ }^{\circ} \mathrm{C}$ and thawed out immediately before running the experiments.

Figure 2 shows the side-chain cleavage region for one of the peptide pairs (peptides BUSM 1 and 2); the lower spectrum is shifted left (by $8.050 \mathrm{Da}$ ) to align the spectra, accounting for the eight deuterium labels. To remove isotopic interferences, the precursor ions were monoisotopically SWIFT isolated before ECD, but the spectra agree with the results from ECD of the nonisotopically isolated samples (data not shown). Abundant side-chain cleavages for BUSM 3 and 4 are apparent and were essentially identical (data not shown), as expected for ECD $[15,36]$. While this side-chain fragment region is very similar between the undeuterated and the glycine deuterated peptides, there are some notable differences marked by dotted lines. The most abundant cluster of side-chain cleavages, which includes the arginine side-chain (59.0483), the aspartic acid (60.0211) side-chain, and the combined loss of $\mathrm{CO}_{2}$ and $\mathrm{NH}_{3}$ (61.0164), showed some evidence of $+\mathrm{D}-\mathrm{H}$ migration to the side-chain before loss. Loss of $\mathrm{H}$. is observed for both the deuterated and nondeuterated peptides, but loss of D. is not observed at all. Furthermore, the loss of $\mathrm{CO}$ is more abundant in the nondeuterated samples than in the deuterated samples. While these effects are subtle, they do indicate that the presence of the $d_{2}$ Gly residues has influenced the isotopic distributions.

While observed in most fragment ions, $\mathrm{d}_{2}$ Gly influenced isotopic pattern changes are more easily ob- 


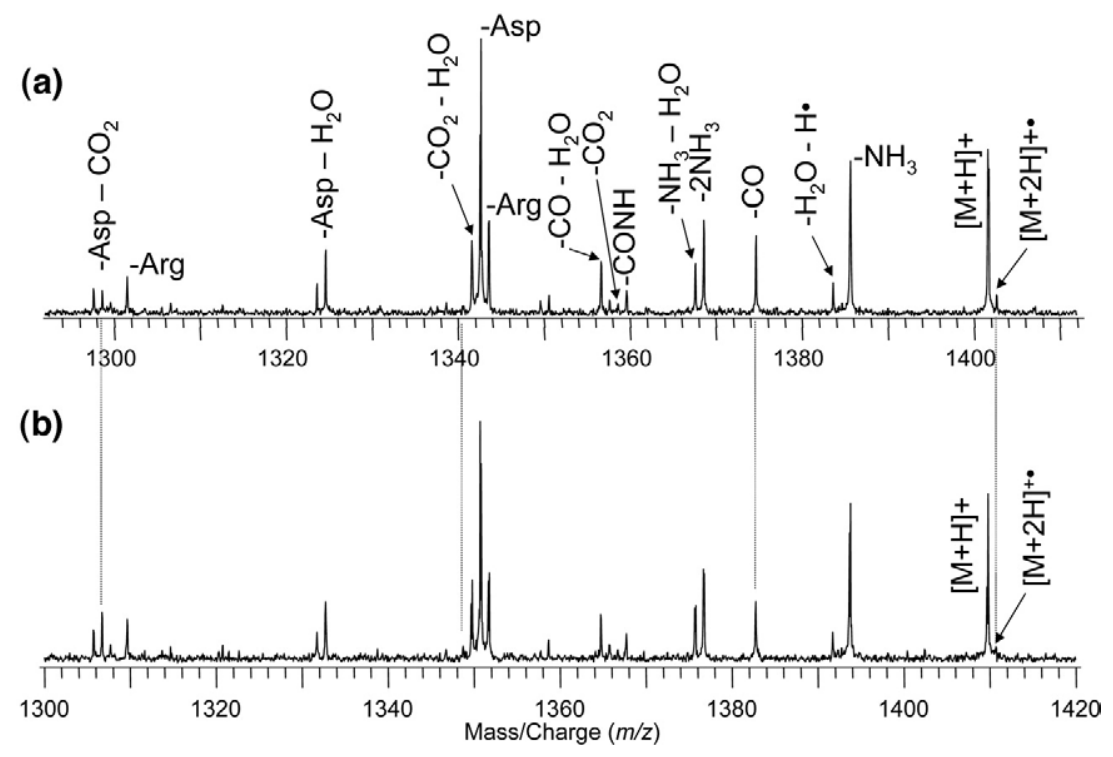

Figure 2. The effect of $\alpha$-carbon hydrogen/deuterium substitution on the side-chain cleavage region of the ECD spectra of the monoisotopically isolated precursor ions of (a) BUSM 1 and (b) BUSM 2.

served in the $\mathrm{c}$ ion series than anywhere else because of their greater abundance. In Figures 3 and 5, each $c_{n}$-ion inset region is plotted as a triplet where the top is the fragment from the undeuterated sample (BUSM 1 and 3) and the middle and lower inset spectra are from the 0.25 and $9 \mathrm{eV}$ ECD spectra, respectively, of the deuterated samples (BUSM 2 and 4; each shifted by $2 \mathrm{Da} /$ glycine for alignment).

Figure 3 shows the $\mathrm{c} / \mathrm{c}$ ions series for the peptide RAAAGADGDGAGADAR (BUSM 1 and 2) from $c_{15}$ down to $C_{5} ; C_{4}-c_{3}$, while detectable, were too low in abundance to generate reliable data, and $c_{5}$ is the smallest $\mathrm{N}$-terminal fragment with a glycine, and hence is the smallest fragment that can show $+\mathrm{H}-\mathrm{D}$ scrambling (that is, deuterium migrating out of the fragment, and hydrogen migrating in). Most of the isotope patterns show substantial changes in the isotopic abundance distribution when comparing the nondeuterated to the deuterated peptides, but the relative isotopic abundance distributions of the 0.25 and $9 \mathrm{eV}$ ECD spectra are remarkably similar in all cases. The most marked isotopic relative abundance distribution differ-
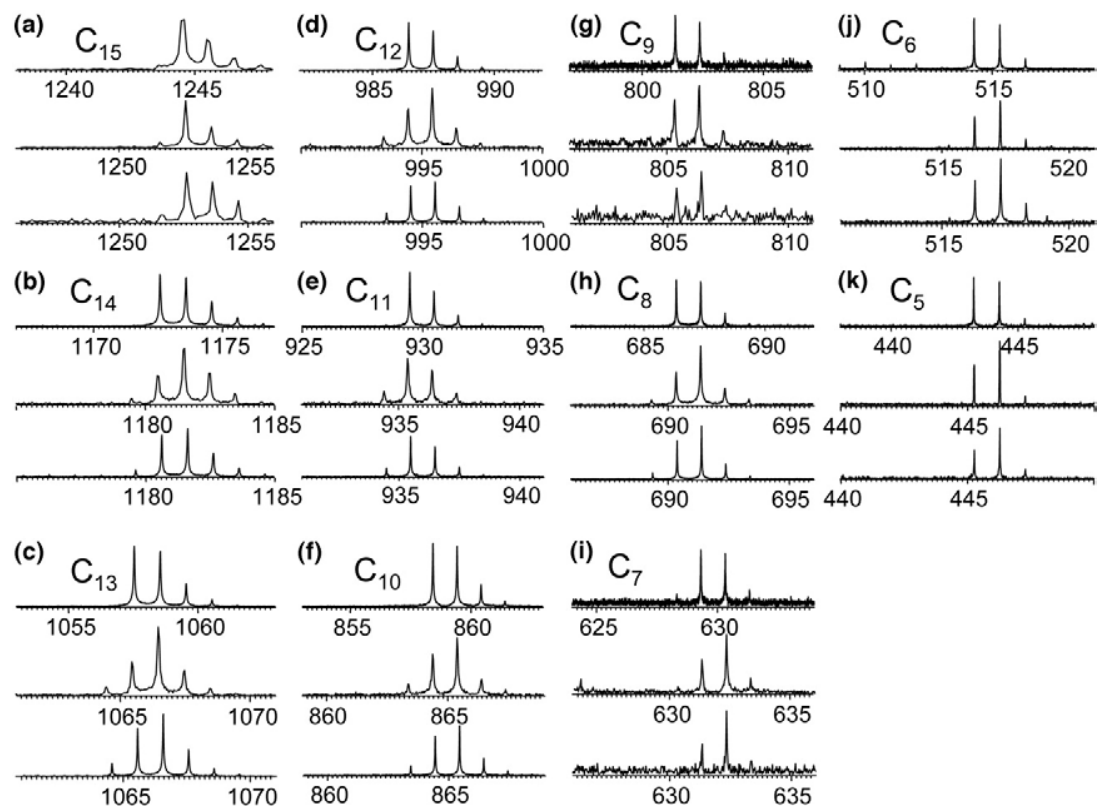

Figure 3. Observed c-ion regions from the ECD spectra for BUSM 1-2. For each panel (a)-(k), top: $0.25 \mathrm{eV}$ ECD of the undeuterated variant (BUSM 1); middle: $0.25 \mathrm{eV}$ ECD of the deuterated variant (BUSM 2); bottom: $9 \mathrm{eV}$ ECD of the deuterated variant (BUSM 2). 

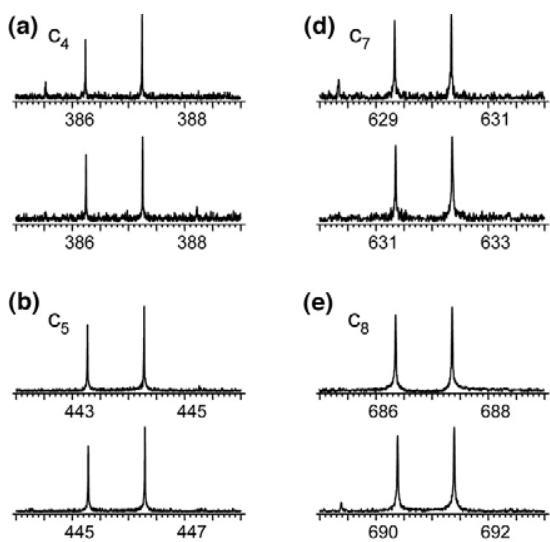

(e)

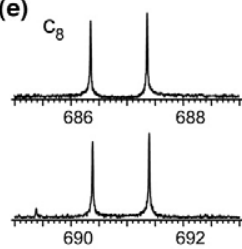

(c)

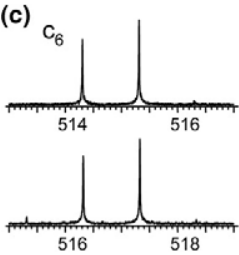

(f)

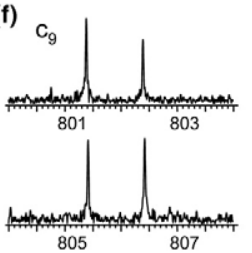

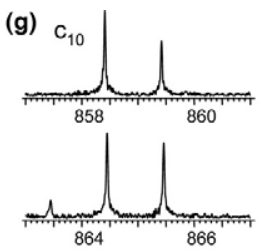

(h)
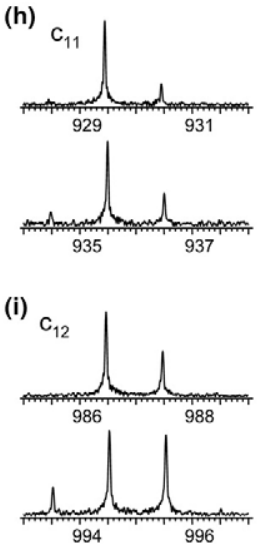

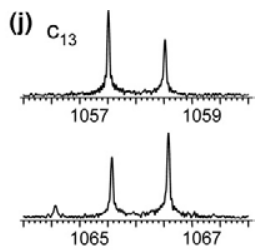

(k)
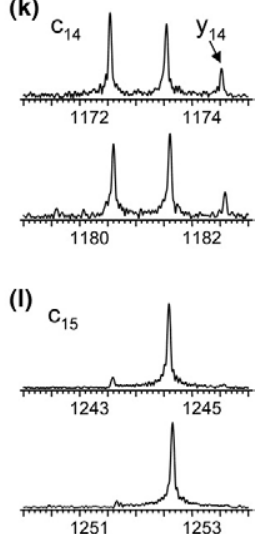

Figure 4. Observed c-ion regions from the $0.8 \mathrm{eV}$ ECD spectra of BUSM 1-2 after monoisotopic isolation of the precursor ion. For each panel (a)-(1), top: the undeuterated variant (BUSM 1); bottom: the deuterated variant (BUSM 2).

ences are apparent in $\mathrm{c}_{14}-\mathrm{c}_{10}$ (Figure $3 \mathrm{~b}-\mathrm{f}$ ), and $\mathrm{c}_{8}$ (Figure $3 \mathrm{~h}$ ). $\mathrm{C}_{9}$ and $\mathrm{C}_{7}$ (Figure $3 \mathrm{~g}$ and i) showed little deuterium scrambling, although the ratio of $c_{n}$ to $c_{n}$ changed. To remove isotopic interferences, Figure 3 was reproduced using monoisotopically isolated precursor ions, and the results are comparable. These results are shown in Figure 4, but without the additional $9 \mathrm{eV}$ data.

Similarly, the peptide RAGADGDADGDAGAAR $c / c$ ions series is plotted from $c_{15}$ down to $c_{4}$ in Figure 5. These spectra also show substantial isotopic pattern differences for most of the peaks, but due to an unfortunate symmetry in the sequence, many of the fragment ions had isotopic distributions that overlapped with other ions; for example $c_{6}$ and $c_{10}$ interfere with $z_{6}$ and $z_{10}$, respectively. This effect makes determination of D-scrambling difficult, and several of these ions are of fairly low signal/noise, which adds substantial error to the quantitation of the D-scrambling for that peptide fragment. Thus, this figure is included to show a second example of isotopic distribution distortions attributable to the presence of $\mathrm{d}_{2} \mathrm{Gly}$, but these data are not discussed further.

The z-ion series (not shown) also reveals some deuterium scrambling, primarily on the $z_{14}$ and $z_{7}$ ions. The remaining $\mathrm{z}$ ions, while observable, are not of sufficient abundance to detect a minor $z_{n}-1$ deuterium scrambling effect. This is also true for the $a_{n}$ ions, which show some clear isotopic pattern shifts, but most of these peaks are not abundant enough to detect $\mathrm{a}_{\mathrm{n}}-1$ peaks.

In general, these spectra show abundant c. ion peaks which, while commonly observed in ECD spectra, are typically not so intense. Several features of these peptides suggest reasons for this. First, the peptides have arginine on both terminal positions to enhance detection of the fragments-which will also enhance detection of the c. ion peaks compared to random peptides. Tryptic peptides often show very low abundance of N-terminal peaks in ECD simply because the C-terminus is always arginine or lysine, thus localizing one charge to the C-terminal amino acid. Second, the data shown here (and discussed below), clearly indicate that migration of the radical to the alpha carbon of glycine is common. These peptides each have four glycines (and several glycine-like alpha carbon positions on the aspartic acid side chains), which are likely to stabilize the radical, thus increasing their abundance in the final spectrum. Third, the gas-phase structure of these peptides is likely to involve multiple hydrogen bonds between the terminal arginine residues and the aspartic acids so that initial electron capture and backbone cleavage would generate a hydrogen bonded complex between the initial c/z fragments, which could then undergo inter-molecular hydrogen abstraction to generate a c. / z complex before formation of the final, observed fragment ions on dissociation of the hydrogenbonded complex. Such a complex agrees with theoretical and experimental data in the literature $[10,20$, 37].

\section{Discussion}

The presence of deuterium labels on glycine has clearly caused distortion in the isotopic patterns of the fragments. There are several possible interpretations to the distortions in the isotopic distributions observed in 

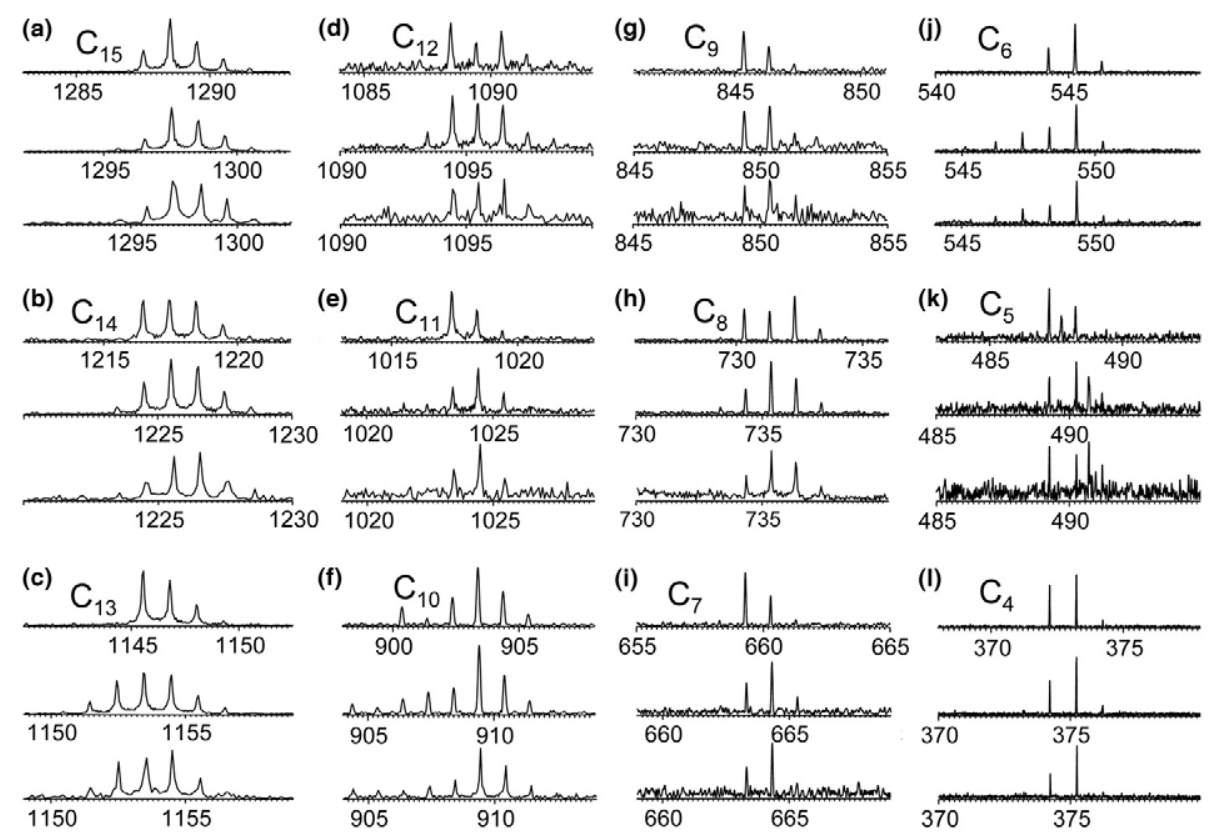

Figure 5. Observed c-ion regions from the ECD spectra for BUSM 3-4. For each panel (a)-(1), top: $0.25 \mathrm{eV}$ ECD of the undeuterated variant (BUSM 3); middle: $0.25 \mathrm{eV}$ ECD of the deuterated variant (BUSM 4); bottom: $9 \mathrm{eV}$ ECD of the deuterated variant (BUSM 4).

Figures 3-5. First, the ion, after electron capture, exists as a long-lived radical cation (possibly as an H-bonded complex between $\mathrm{C}$-terminal and N-terminal fragments), which undergoes one or more hydrogen abstraction events before final dissociation, generating the c-1 peaks that are observed only in the deuterated spectra. Second, the c. ions from the deuterated peptide undergo $\mathrm{H}$. loss to a much higher extent than the undeuterated peptides. Third, the even electron $\mathrm{c}_{\mathrm{n}}{ }^{+}$ ions could lose D. to form the radical cation $\left[\mathrm{c}_{\mathrm{n}}-\mathrm{D}\right]^{+}$. These three interpretations will be discussed below. However, it is important to remember that the difference between these structures is deuteration of the nonexchangeable and nonhydrogen bonded alpha carbon of glycine residues in the sequences.

The first interpretation of the c-1 peaks is shown in Scheme 1. In this scheme, the initial electron capture event has caused a backbone bond cleavage forming the typical $\mathrm{c}$ and $\mathrm{z}$. ion pair that remains bound by a hydrogen bond [10, 20,37]. This complex is a long-lived radical intermediate in the electron capture dissociation mechanism. The terminal radical on the $\mathrm{z} \cdot$ ion abstracts a hydrogen (or deuterium) from a more stable site, in this case the alpha carbon of a glycine on the c-fragment thus forming a c. and $\mathrm{z}$ ion pair, but with the deuterium atoms scrambled. Sometime later, the hydrogen bond breaks, and the two fragments are free to be detected independently. This mechanism would be driven by the stability of the glycine alpha carbon radical product. Scheme 2 is essentially a variant of this same mechanism, but involves intra- rather than inter-fragment deuterium scrambling.

The second interpretation of the c--1 peaks is that they could be formed by loss of $\mathrm{H}$. from the c. ion. Such losses are not uncommon in ECD spectra. However, these peaks are present only in the ECD spectra of the deuterated peptides and not in the spectra of the undeuterated peptides. Perhaps the presence of the $\mathrm{d}_{2}$ Gly residues effectively destabilizes the $\mathrm{c}$. radical by preventing (via a kinetic isotope effect, see below) the radical from migrating to the relatively stable radical position at the alpha carbon of glycine. However, this interpretation seems less likely attributable to the absence of c-1 peaks in the nondeuterated peptides.

The third possible interpretation of the c- -1 peaks is that it is caused by loss of D. from the even electron c peak. This interpretation would imply formation of an unstable radical cation, $\left[c_{n}-D\right]^{+}$, from the relatively

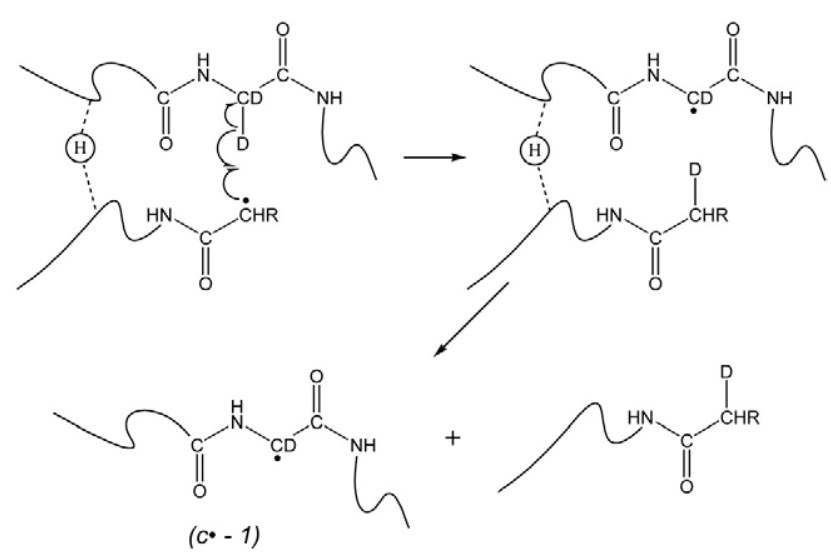

Scheme 1. One possible mechanism to describe observation of $(+\mathrm{H}-\mathrm{D})$ scrambling c. fragments. 
<smiles>[R]C(CCC)C(=O)NC([R2])C(=O)NC(=O)C(=O)CCCC</smiles>

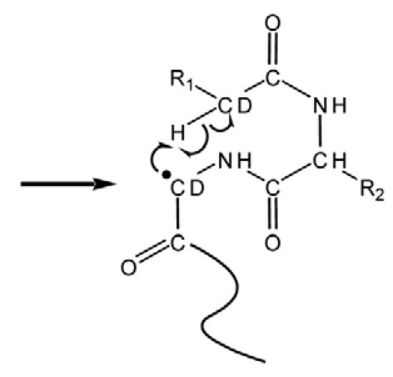<smiles>[R]OC(=O)NC(=O)C([R2])NC(=O)C(=O)CCCC</smiles><smiles>[R]C1NC(=O)C([R])NC1=O</smiles>

Scheme 2. A second possible mechanism to describe observation of $(+H-D)$ scrambling fragments.

stable even electron $c_{n}$ ion which seems to be the least likely scenario.

Theoretically, the first and second interpretations can be distinguished by mass, but the first and third interpretations result in isobaric fragment ions. The $\left[\mathrm{c}_{\mathrm{n}}-\mathrm{D}+\mathrm{H}\right]^{+\cdot}$ scrambled species would have a mass that is $2.0140-1.0078=1.0062$ Da lower than the $\mathrm{c}_{\mathrm{n}}{ }^{+\cdot}$ ion, while the $\left[\mathrm{c}_{\mathrm{n}}-\mathrm{H}\right]^{+}$ion would have a mass that is $1.0078 \mathrm{Da}$ lower than the $\mathrm{c}_{\mathrm{n}}{ }^{+\cdot}$ ion. This mass difference of $1.6 \mathrm{mDa}$ can theoretically be distinguished provided that the mass spectrometer can achieve at least $900 \mathrm{k}$ resolving power (FWHM) for the molecular ion or somewhat lower for fragment ions. In practice, because of the peak coalescence effect in Fourier transform mass spectrometry [38, 39], 2-3× that resolving power (or 2-3 million) would likely be required. In the reported experiments, such resolving power was not achieved.

Hydrogen abstraction, and hence deuterium scrambling, is expected to be a slow reaction (microseconds) due to the need for conformational change of the gas-phase peptide ion to bring the radical close to the hydrogen before reaction. The presence of the deuterium labels resulted in substantial isotopic pattern distortions, suggesting that the radical migrates to the $\alpha$-carbon of glycine during ECD. However the radical migration, when it occurs, is likely to occur several (or many) times before cleavage. The data, primarily in Figures 3-5, show that H/D scrambling is occurring from the $\alpha$-carbon positions. As discussed above, most likely interpretation of these data is that many of the observed ECD fragments are formed from a radical intermediate complex which has undergone one or more radical rearrangements via hydrogen abstraction from the alpha carbon positions.

The side-chain loss region showed some subtle isotopic pattern differences between Gly and $\mathrm{d}_{2}$ Gly containing peptide fragments, but it remains unclear why $\mathrm{CO}$ losses would be H/D dependent. A better experiment for observation of side-chain scrambling would be to use peptides with some deuterium labeled side chains, but no other deuterium labels. Such an experiment would allow rough quantitation of the ratio of side chain to backbone hydrogen abstraction in a longlived peptide radical cation.

\section{Energetics Effects}

CAD experiments performed in Q2 [26] in transit to the ICR cell at $\sim 23 \mathrm{eV}$ lab-frame collision energy and SORI-CAD experiments in the ICR cell at $\sim 0.1 \mathrm{eV} /$ collision on average were used to probe the scrambling of deuterium under ergodic fragmentation. The results are plotted in Figure 6 and compared with the ECD data. The $b$ and $c$ ions are plotted together (Figure 6a) for comparison in terms of the approximate number of exchangeable hydrogens. This figure plots the ratio of the ion abundances (from Figures 3 and 4) corrected for under-deuteration as discussed above. However, at this point it is not possible to distinguish $(+\mathrm{H}-\mathrm{D})$ scrambling on the even electron ion from the odd electron ion abundance or $+\mathrm{D}-\mathrm{H}$ scrambling on the radical fragment from the even electron ion abundance. Thus, these scrambling ratios may be an underestimate.

Deuterium scrambling of even electron molecular ions under CAD conditions is well known [40-45], and has been explored in the same manner as was done here with D's located on the alpha carbon positions [46]. Under energetic (23 eV) CAD conditions for BUSM 2 (Figure 6a), scrambling occurred in most fragment ion isotopic distributions, with the notable exception of the extremely labile $b_{9} / y_{7}$ and $b_{7} / y_{9}$ complementary ion pairs. Thus, in CAD, these labile fragments undergo dissociation before scrambling, but the rest of the fragments have time for exchange before cleavage. ECD, however, generates odd electron radical cations, which show a different internal energy dependence. ECD with the cell at elevated temperature by being in direct physical contact with the electron gun and ECD with the electron gun pulled back generated spectra that show similar levels of deuterium scrambling to each other with a slight (and expected) preference for smaller fragments in the "elevated temperature" ECD experiment, presumably due to secondary backbone cleavages.

The CAD and ECD results plotted in Figure 6a represent data from fragmentation that occurred in different regions of the instrument, which could result in different internal vibrational energy distributions in the precursor ions and perhaps influence the scrambling results. To test this possibility, SORI-CAD $[47,48]$ experiments were performed in the ICR cell with the vacuum chamber at 25 and $150{ }^{\circ} \mathrm{C}$, with several seconds 


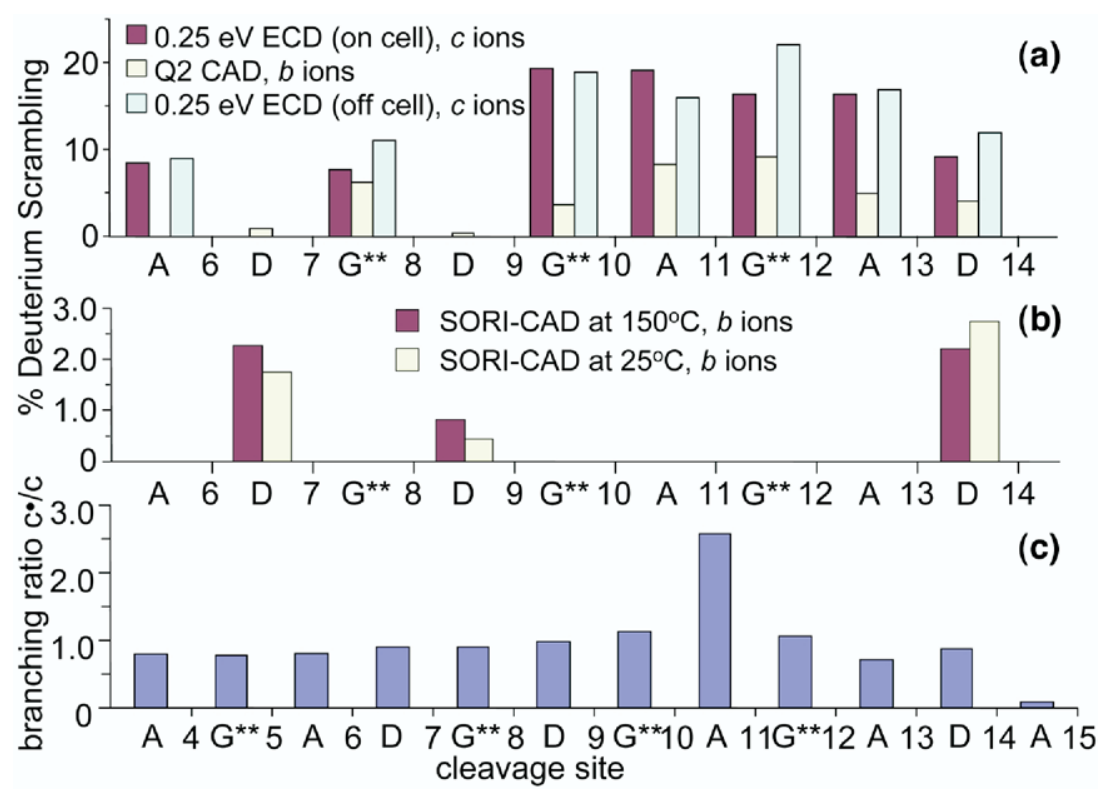

Figure 6. Site specific $(+\mathrm{H}-\mathrm{D})$ scrambling ratios for BUSM 2. (a) comparison of $0.25 \mathrm{eV}$ ECD with the electron gun mounted on the cell or $\sim 15 \mathrm{~cm}$ back with CAD Experiment; (b) deuterium scrambling under SORI-CAD at two different temperatures; (c) branching ratio of $\mathrm{c}$. ion formation over c ion formation (from Figure 4).

of equilibration time before fragmentation (equilibration of peptide internal vibrational energy with background IR radiation typically occurs on a msec time scale [49]). SORI-CAD is well known to "slow heat" molecules so that selective cleavage of the most labile bonds is preferred. Since the BUSM 1 peptide has three aspartic acid residues, and the most likely bond to break is the bond C-terminal to aspartic acid $[50,51]$, only $b_{7}$, $b_{9}, b_{14}, y_{7}$, and $y_{9}$ were observed ( $y_{2}$ is below the mass range of the experiment). However, the SORI-CAD and Q2-CAD experiments effectively bracketed the ECD experiments both in terms of total energy available and duration. While the IR radiative heating of the vacuum chamber clearly influenced the SORI-CAD fragment distributions to favor smaller fragments, none of the deuterium exchange levels in the SORI-CAD experiments exceeded 3\%. $0.1 \mathrm{eV} /$ collision SORI-CAD experiments, even at $150{ }^{\circ} \mathrm{C}$, resulted in an order of magnitude less deuterium scrambling than room temperature ECD experiments with a total available energy of $\sim 6 \mathrm{eV}$ [1].

In spite of the greater energy available, the CAD spectra, both $23 \mathrm{eV}$ Q2-CAD and $0.1 \mathrm{eV} /$ collision SORI$\mathrm{CAD}$, show much less scrambling than the ECD spectra. Furthermore, the $0.25 \mathrm{eV}$ ECD spectra are remarkably similar to the $9 \mathrm{eV}$ ECD spectra (Figures 3 and 4), indicating that, in this energy range, D scrambling is not highly dependent on the internal vibrational energy. Thus, for the radical cations produced by ECD, scrambling must occur with transition-state barriers much lower than the redistributed vibrational energy of the molecule, which agrees with existing calculations in the literature [10, 11, 23, 24, 52].

Figure $6 c$ shows the branching ratios of the $c_{n}$ and $c_{n}$. ions. These data are plotted from the $c_{n}$ and $c_{n}$. abundances of the ECD spectra of the monoisotopically isolated BUSM 2 peptide molecular ion (see Figure 4). Except for $\mathrm{c}_{11}$ and $\mathrm{c}_{15}$, most of the branching ratios were $\sim 1$. $C_{15}$ showed almost no $c_{15}$. formation, implying that the terminal arginine residue prefers to leave as a neutral radical species. Likewise, the $c_{11}$ peak showed strong preference $\left(\sim 3\right.$-fold) for the formation of $c_{11}$. The reasons for these strong deviations from the average are unclear, but they are likely to be related to the gas-phase folding structure of these peptides.

\section{Kinetic Isotope Effect}

In any comparison of hydrogen versus deuterium labeled reactions, it is important to consider a kinetic isotope effect in the fragmentation. If several reaction channels are available, and at least one of these channels involves $\mathrm{H}$ versus D loss or migration, the KI effect can greatly influence the abundances of the reaction products, sometimes an order of magnitude or more in abundance [53, 54]. In a free radical cascade, if the reaction channel quenches the radical or allows a neutral radical loss, the reaction is over. The KI effect, along with the fact that the charge sites in these peptides are protons, not deuterons, is one possible reason why $\mathrm{H}$. loss is observed in the charge reduced radical cation $[\mathrm{M}+2 \mathrm{H}]^{+*}$, but $\mathrm{D}$. loss is not. Furthermore, the abundance change in the - $\mathrm{CO}$ sidechain loss (Figure 3) points to the presence of a KI effect, although the mechanism of this reaction is unclear at this time. However, if the reaction channel chosen does not result in the loss of a radical cleavage fragment, the radical is still present in the molecule and can initiate further 
reactions. For inter- or intra-molecular hydrogen (deuterium) abstraction reactions (as in Schemes $\mathbf{1}$ and 2), this is the case. In spite of the KI effect that must be present in $\alpha$-carbon hydrogen abstraction reactions, the long time frame of the FTICR experiments (seconds) appears to partially overcome the effect so that deuteriums are allowed to migrate. Furthermore, if the KI effect is partially suppressing the deuterium migration relative to hydrogen migration, as would be expected, the migration percentages reported above must be considered underestimates.

\section{Ratio of Primary to Secondary Cleavages}

This experiment was originally designed to attempt to distinguish primary from secondary cleavages, based on the premise that secondary cleavages proceeded via a hydrogen (or deuterium) abstraction from the alpha carbon site of glycine. If the peptide fragmentation proceeded via the described mechanism [14], deuterium scrambling would be the inevitable result. The data shown support this view. However, certain fragments show remarkably little scrambling (notably, those c-terminal to aspartic acid), while others show extensive scrambling (those adjacent to glycine residues). Could it be that the former are primary fragments while the latter are the result of secondary backbone cleavage? Based on the results by Mihalca et al. [55] and Leymarie et al. [14], this seems likely.

Thus, a reasonable explanation for the observed scrambling pattern is that while the C-terminal aspartic acid cleavages proceed rapidly (possibly nonergodically), the remaining fragments are apparently formed from both a statistically determined rapid backbone cleavage and a series of slow, free radical rearrangements. These data show that a substantial fraction of these cleavages are formed after free-radical migration. (Note that radical migration which produces net-zero deuterium scrambling cannot be differentiated from direct, rapid backbone cleavage on the $\sim 1 \mathrm{~s}$ time frame used in these experiments.) Since only 4/11 (BUSM 2, Figures 3 and 4 ) and 4/9 (BUSM 4, Figure 5, neglecting distributions with interferences) detected cleavages showed effectively no deuterium scrambling, this implies that at least half of the ECD ions in these peptides are actually formed from secondary cleavages or as the products of long-lived radical intermediates.

\section{Ergodicity}

The data shown here, unfortunately, does not resolve the debate about the ergodicity of ECD primary fragments [1, 11] because all of the normal ECD fragments are present. The aspartic acid cleavages at $c_{7}$ and $c_{9}$ showed little or no deuterium scrambling, which is consistent with a nonergodic primary dissociation pathway. However, deuterium scrambling is a very slow probe and is likely to occur on a microsecond time scale, which is much too slow to rule out intramolecular vibrational redistribution (which occurs on a picosecond time scale) [22] of the initial electron capture energy before fragmentation. The presence of the deuterium scrambling peaks elsewhere does support a pathway that involves a long-lived radical ion complex in which radical migration via $\mathrm{H}$ (or $\mathrm{D}$ ) abstraction occurs. Combined with previous data [14], this H/D scrambling data suggests the existence of at least two mechanisms; one slow, one fast.

Finally, although ECD related $+\mathrm{H}-\mathrm{D}$ scrambling percentages are noted to be up to $\sim 20 \%$ in Figures 3-6, this value is likely to be a substantial underestimation of the extent of radical migration in these peptides. First, the kinetic isotope effect will result in suppression of radicalinduced abstraction of D. versus H. Without D. abstraction, the radical based scrambling of hydrogen atoms is unobservable. Second, until ultra-high-resolution (2-3 M, FWHM for peptides of this size) experiments can be performed, it is impossible to observe $+\mathrm{D}-\mathrm{H}$ scrambling from the $c_{n}$. ions or $+H-D$ scrambling from the $c_{n}$ ions due to peak overlap with the unscrambled $c_{n}$ and $c_{n}$. ions, respectively. Both of these events (which are expected) would add to the extent of scrambling. Thus, this underestimation of the scrambling suggests that the real scrambling levels could be as much as an order of magnitude higher.

\section{Conclusions}

In an experiment designed to test the electron capture dissociation mechanism in linear peptides, deuterium scrambling via free-radical abstraction of alpha carbon deuterium labels was observed. Distortion of the isotopic distributions of the fragment ions was observed because of the presence of $\mathrm{d}_{2} \mathrm{Gly}$. Furthermore, although the Q2 CAD experiment, at $23 \mathrm{eV}$ collision energy, provided more internal vibrational energy than the ECD spectra (with 0.2-9 eV electron kinetic energy depending on the experiment and $\sim 6 \mathrm{eV}$ of electron-ion recombination energy [1]), the CAD spectrum showed lower D-scrambling than did the ECD spectrum. This experiment demonstrates that many of the expected $\mathrm{c} / \mathrm{z}$ ions for ECD are products of long-lived radical intermediates that migrate via the abstraction of $\mathrm{H}$ (or D) from the $\alpha$-carbon of glycine residues.

\section{Acknowledgments}

The authors thank Kathrin Breuker, Cathy Costello, Ron Heeren, Fred McLafferty, Frank Turecek, and Roman Zubarev for helpful discussions. The work was supported by NIH/NCRR P41RR10888, NIH/NHLBI NO1HV28178, by a collaborative research agreement with MDS/Sciex, and by the donors of The American Chemical Society Petroleum Research Fund.

\section{References}

1. Zubarev, R. A.; Kelleher, N. L.; McLafferty, F. W. Electron capture dissociation of multiply charged protein cations-a nonergodic process. J. Am. Chem. Soc. 1998, 120, 3265-3266.

2. Zubarev, R. A. Kruger, N. A.; Fridriksson, E. K. Lewis, M. A. Horn, D. M. Carpenter, B. K.; McLafferty, F. W. Electron capture dissociation of gaseous multiply-charged proteins is favored at disulfide bonds and other sites of high hydrogen atom affinity. J. Am. Chem. Soc. 1999, 121, 2857-2862. 
3. McLafferty, F. W.; Turecek, F. Interpretation of mass spectra, 4th ed.; University Science Books: Mill Valley, CA, 1993.

4. Horn, D. M.; Breuker, K.; Frank, A. J.; McLafferty, F. W. Kinetic intermediates in the folding of gaseous protein ions characterized by electron capture dissociation mass spectrometry. J. Am. Chem. Soc. 2001, 123, 9792-9799.

5. Oh, H.; Breuker, K.; Sze, S. K.; Ge, Y.; Carpenter, B. K.; McLafferty, F. W Secondary and tertiary structures of gaseous protein ions characterized by electron capture dissociation mass spectrometry and photofragment spectroscopy. Proc. Nat. Acad. Sci. U.S.A. 2002, 99, 15863-15868.

6. Kelleher, R. L.; Zubarev, R. A.; Bush, K.; Furie, B.; Furie, B. C. McLafferty, F. W.; Walsh, C. T. Localization of labile posttranslational modifications by electron capture dissociation: The case of gammacarboxyglutamic acid. Anal. Chem. 1999, 71, 4250-4253.

7. Mirgorodskaya, E.; Roepstorff, P.; Zubarev, R. A. Localization of o-glycosylation sites in peptides by electron capture dissociation in a Fourier transform mass spectrometer. Anal. Chem. 1999, 71, 4431-4436.

8. Hakansson, K.; Cooper, H. J.; Emmett, M. R.; Costello, C. E.; Marshall, A. G.; Nilsson, C. L. Electron capture dissociation and infrared multiphoton dissociation MS/MS of an n-glycosylated tryptic peptide to yield complementary sequence information. Anal. Chem. 2001, 73, 4530-4536.

9. Mormann, M.; Macek, B.; Peredo, A. G. D.; Hofsteenge, J.; PeterKatalinic, J. Structural studies on protein o-fucosylation by electron capture dissociation. Int. J. Mass Spectrom. 2004, 234, 11-21.

10. Turecek, F.; Syrstad, E. A. Mechanism and energetics of intramolecular hydrogen transfer in amide and peptide radicals and cation-radicals. J. Am. Chem. Soc. 2003, 125, 3353-3369.

11. Turecek, F. N-c $\alpha$ bond dissociation energies and kinetics in amide and peptide radicals. Is the dissociation a nonergodic process? J. Am. Chem. Soc. 2003, 125, 5954-5963.

12. Syrstad, E. A.; Stephens, D. D.; Turecek, F. Hydrogen atom adducts to the amide bond. Generation and energetics of amide radicals in the gas phase. J. Phys. Chem. A 2003, 107, 115-126.

13. Turecek, F.; Syrstad, E. A.; Seymour, J. L.; Chen, X. H.; Yao, C. X Peptide cation-radicals. A computational study of the competition between peptide $n-c \alpha$, bond cleavage, and loss of the side chain in the $\left[\text { GlyPhe- } \mathrm{NH}_{2}+2 \mathrm{H}\right]^{+\cdot}$ cation-radical. J. Mass Spectrom. 2003, 38, 1093 1104.

14. Leymarie, N.; Costello, C. E.; O'Connor, P. B. Electron capture dissociation initiates a free radical reaction cascade. I. Am Chem Soc. 2003, 125, 8949-8958.

15. Cooper, H. J.; Hudgins, R. R.; Hakansson, K.; Marshall, A. G. Characterization of amino acid side chain losses in electron capture dissociation. J. Am. Soc. Mass Spectrom. 2002, 13, 241-249.

16. Cooper, H. J.; Hudgins, R. R.; Hakansson, K.; Marshall, A. G. Secondary fragmentation of linear peptides in electron capture dissociation. Int. J. Mass Spectrom. Ion Processes 2003, 228, 723-728.

17. Cooper, H. J.; Hudgins, R. R.; Marshall, A. G. Electron capture dissociation Fourier transform ion cyclotron resonance mass spectrometry of cyclodepsipeptides, branched peptides, and $\varepsilon$-peptides. Int. I. Mass Spectrom. Ion Processes 2004, 234, 23-35.

18. Kjeldsen, F.; Haselmann, K. F.; Budnik, B. A.; Jensen, F.; Zubarev, R. A Dissociative capture of hot (3-13 eV) electrons by polypeptide polycations: An efficient process accompanied by secondary fragmentation. Chem. Phys. Lett. 2002, 356, 201-206.

19. Hodyss, R.; Cox, H. A.; Beauchamp, J. L. Bioconjugates for tunable peptide fragmentation: Free radical initiated peptide sequencing (FRIPS). J. Am. Chem. Soc. 2005, 127, 12436-12437.

20. Fung, Y. M. E.; Chan, T.-W. D. Experimental and theoretical investigations of the loss of amino acid side chains in electron capture dissociation of model peptides. J. Am. Soc. Mass Spectrom. 2005, 16, 1523-1535.

21. Hu, Y. J.; Hadas, B.; Davidovitz, M.; Balta, B.; Lifshitz, C. Does IVR take place prior to peptide ion dissociation? J. Phys. Chem. A 2003, 107, 6507-6514.

22. Nummela, J. A.; Carpenter, B. K. Nonstatistical dynamics in deep potential wells: A quasiclassical trajectory study of methyl loss from the acetone radical cation. J. Am. Chem. Soc. 2002, 124, 8512-8513.

23. Rauk, A.; Yu, D.; Armstrong, D. A. Oxidative damage to and by cysteine in proteins: An ab initio study of the radical structures, $c-h, s-h$, and $\mathrm{c}-\mathrm{c}$ bond dissociation energies, and transition structures for $\mathrm{h}$ abstraction by thiyl radicals. J. Am. Chem. Soc. 1998, 120, 8848-8855.

24. Rauk, A.; Yu, D.; Taylor, J.; Shustov, G. V.; Block, D. A.; Armstrong, D. A. Effects of structure on $\alpha \mathrm{c}-\mathrm{h}$ bond enthalpies of amino acid residues: Relevance to $H$ transfers in enzyme mechanisms and in protein oxidation. Biochemistry 1999, 38, 9089-9096.

25. Jebanathirajah, J. A.; Pittman, J. L.; Thomson, B. A.; Budnik, B. A.; Kaur, P.; Rape, M.; Kirschner, M.; Costello, C. E.; O'Connor, P. B. Characterization of a new qQq-FTICR mass spectrometer for post-translational modification analysis and top-down tandem mass spectrometry of whole proteins. J. Am. Soc. Mass Spectrom. 2005, 16, 1985-1999.

26. O'Connor, P. B.; Pittman, J. L.; Thomson, B. A.; Budnik, B. A.; Cournoyer, J. C.; Jebanathirajah, J.; Lin, C.; Moyer, S. A new hybrid electrospray Fourier transform mass spectrometer: Design and performance characteristics. Rapid Commun. Mass Spectrom. 2006, 20, 259-266.

27. Valaskovic, G. A.; Kelleher, N. L.; Little, D. P.; Aaserud, D. J.; McLaf ferty, F. W. Attomole-sensitivity electrospray source for large-molecule mass spectrometry. Anal. Chem. 1995, 67, 3802-3805.

28. Tsybin, Y. O.; Hakansson, P.; Budnik, B. A.; Haselmann, K. F.; Kjeldsen, F.; Gorshkov, M.; Zubarev, R. A. Improved low-energy electron injection systems for high rate electron capture dissociation in Fourier transform ion cyclotron resonance mass spectrometry. Rapid Commun. Mass Spectrom. 2001, 15, 1849-1854

29. Roepstorff, P.; Fohlman, J. Proposal for a common nomenclature for sequence ions in mass spectra of peptides. Biomed. Mass Spectrom. 1984, 11, 601.

30. Biemann, K. Sequencing of peptides by tandem mass spectrometry and high-energy collision-induced dissociation. Methods Enzymol. 1990, 193, $455-479$.

31. Cournoyer, J. J.; Pittman, J. L.; Ivleva, V. B.; Fallows, E.; Waskell, L.; Costello, C. E.; O'Connor, P. B. Deamidation: Differentiation of aspartyl from isoaspartyl products in peptides by electron capture dissociation. Protein Sci. 2005, 14, 452-463.

32. O'Connor, P. B.; Cournoyer, J. J.; Pitteri, S. J.; Chrisman, P. A.; Mcluckey, S. A. Differentiation of aspartic and isoaspartic acids using electron transfer dissociation. J. Am. Soc. Mass Spectrom. 2006, 17, 15-19.

33. Cournoyer, J. J., Lin, C., O'Connor, P. B. Detecting deamidation products in proteins by electron capture dissociation. Anal. Chem. 2006, in press.

34. Dunbar, R. C.; McMahon, T. B. Activation of unimolecular reactions by ambient blackbody radiation. Science 1998, 279, 194-197.

35. Horn, D. M.; Ge, Y.; McLafferty, F. W. Activated ion electron capture dissociation for mass spectral sequencing of larger $(42 \mathrm{kDa})$ proteins. Anal. Chem. 2000, 72, 4778-4784.

36. Haselmann, K. F.; Budnik, B. A.; Kjeldsen, F.; Polfer, N. C.; Zubarev, R. A. Can the $(\mathrm{m} \cdot \mathrm{-x})$ region in electron capture dissociation provide reliable information on the amino acid composition of polypeptides? Eur. J. Mass Spectrom. 2002, 8, 461-469.

37. McLafferty, F. W.; Horn, D. M.; Breuker, K.; Ge, Y.; Lewis, M. A.; Cerda, B.; Zubarev, R. A.; Carpenter, B. K. Electron capture dissociation of gaseous multiply charged ions by Fourier-transform ion cyclotron resonance. J. Am. Soc. Mass Spectrom. 2001, 12, 245-249.

38. Chen, S.-P.; Comisarow, M. B. Modeling coulomb effects in FTICRMS by charged disks and charged cylinders. Rapid Commun. Mass Spectrom. $1992,6,1-3$.

39. Stults, J. T. Minimizing peak coalescence: High resolution separation of isotope peaks in partially deamidated peptides by matrix assisted laser desorption/ionization Fourier transform ion cyclotron resonance mass spectrometry. Anal. Chem. 1997, 69, 1815-1819.

40. Hagman, C.; Hakansson, P.; Buijs, J.; Hakansson, K. Inter-molecular migration during collisional activation monitored by hydrogen/deuterium exchange FTICR tandem mass spectrometry. J. Am. Soc. Mass Spectrom. 2004, 15, 639-646.

41. Buijs, J.; Hagman, C.; Hakansson, K.; Richter, J. H.; Hakansson, P.; Oscarsson, S. Inter- and intra-molecular migration of peptide amide hydrogens during electrospray ionization. J. Am. Soc. Mass Spectrom. 2001, 12, 410-419.

42. Johnson, R. S.; Krylov, D.; Walsh, K. A. Proton mobility within electrosprayed peptide ions. J. Mass Spectrom. 1995, 30, 386-387.

43. Reid, G. E.; Simpson, R. J.; O'Hair, R. A. J. Probing the fragmentation reactions of protonated glycine oligomers via multistage mass spectrometry and gas phase ion molecule hydrogen/deuterium exchange. Int. J. Mass Spectrom. 1999, 190/191, 209-230.

44. Mueller, D. R.; Eckersley, M.; Richter, W. J. Hydrogen transfer reactions in the formation of " $y+2$ " sequence ions from protonated peptides. Org. Mass Spectrom. 1988, 23, 217-222.

45. Demmers, J. A. A.; Rijkers, J. T. S.; Haverkamp, J.; Killian, J. A.; Heck, A. J. R. Factors affecting gas-phase deuterium scrambling in peptide ions and their implications for protein structure determination. J. Am. Chem. Soc. 2002, 124, 11191-11198.

46. Kenny, P. T. M.; Nomoto, K.; Orlando, R. Fragmentation studies of peptides: The formation of y ions. Rapid Commun. Mass Spectrom. 1992, 6, 95-97.

47. Gauthier, J. W.; Trautman, T. R.; Jacobson, D. B. Sustained off-resonance irradiation for collision-activated dissociation involving Fourier transform mass spectrometry. Collision-activated dissociation technique that emulates infrared multiphoton dissociation. Anal. Chim. Acta 1991, 246, 211-225.

48. Mirgorodskaya, E.; O'Connor, P. B.; Costello, C. E. A general method for precalculation of parameters for sustained off resonance irradiation/collisioninduced dissociation. J. Am. Soc. Mass Spectrom. 2002, 13, 318-324.

49. Dunbar, R. C. Infrared radiative cooling of gas-phase ions. Mass Spectrom. Rev. 1992, 11, 309-339.

50. Tsaprailis, G.; Somogyi, A.; Nikolaev, E. N.; Wysocki, V. H. Refining the model for selective cleavage at acidic residues in arginine-containing protonated peptides. Int. J. Mass Spectrom. Ion Processes 2000, 196, 467-479.

51. Gu, C. G.; Tsaprailis, G.; Breci, L.; Wysocki, V. H. Selective gas-phase cleavage at the peptide bond terminal to aspartic acid in fixed-charge derivatives of asp-containing peptides. Anal. Chem. 2000, 72, 5804-5813.

52. Rauk, A.; Yu, D.; Armstrong, D. A. Toward site specificity of oxidative damage in proteins: $\mathrm{C}-\mathrm{h}$ and $\mathrm{c}-\mathrm{c}$ bond dissociation energies and reduction potentials of the radicals of alanine, serine, and threonine residues-an ab initio study. J. Am. Chem. Soc. 1997, 119, 208-217.

53. Derrick, P. J. Isotope effects in fragmentation. Mass Spectrom. Rev. 1983, 2, 285-298.

54. Lehman, T. A. Isotope effects in the bimolecular reactions of gaseous ions. Concepts, techniques, and reactions. Mass Spectrom. Rev. 1995, 14, 353-382.

55. Mihalca, R.; Kleinnijenhuis, A. J.; McDonnell, L. A.; Heck, A. J. R.; Heeren, R. M. A. Electron capture dissociation at low temperatures reveals selective dissociations. J. Am. Soc. Mass Spectrom. 2004, 15, 1869-1873. 